\title{
LA MARCA ONLINE COMO PARTE DE LA COMUNICACIÓN INTEGRAL. MANEJO DE MARCAS ECUATORIANAS EN LAS REDES SOCIALES
}

\author{
Paladines Galarza, Fanny Yolanda ${ }^{1}$ \\ Granda Tandazo, Carlos Vladimir ${ }^{2}$ \\ Departamento de Ciencias de la Comunicación \\ Universidad Técnica Particular de Loja (UTPL) \\ fypaladines@utpl.edu.ec \\ cwgranda@utpl.edu.ec
}

Material original autorizado para su primera publicación en la revista académica REDMARKA. Revista Digital de Marketing Aplicado.

https://doi.org/10.17979/redma.2013.01.010.4758

Recibido: 12 Marzo 2013

Aceptado 13 Junio 2013

\section{Resumen}

El uso de Internet en la promoción y publicidad tiene un amplio alcance. Como señala Araceli Castelló (2010, p. 14-15) un ejemplo claro es la demanda por parte de los anunciantes, de espacios como el ranking de palabras más

1 Licenciada en Publicidad por la Universidad Tecnológica Equinoccial de Quito; Máster en Gestión y Dirección de Marketing de la Escuela de Organización Industrial de Madrid; y tiene un doctorado (Ph.D.) en Comunicación y Periodismo por la Universidad Santiago de Compostela. Docente investigadora principal en Publicidad, Marketing, Comunicación Organizacional y Cultura Corporativa de las Titulaciones de Comunicación Social, Relaciones Públicas y Asistencia Gerencial. Dirigió por 4 años la Gerencia de Marketing y Publicidad de la UTPL. Actualmente pertenece al Departamento de Ciencias de la Comunicación en la línea de investigación de la Sección Departamental de Comunicación Organizacional.

2 Licenciado en Mercadotecnia por la Universidad Tecnológica Equinoccial de Quito; Máster en Gestión y Dirección de Marketing de la Escuela de Organización Industrial de Madrid; es candidato a Doctor (Ph.D.) en Comunicación Social y Periodismo por la Universidad Santiago de Compostela. Docente investigador universitario desde hace 12 años, en las áreas de Marketing, Investigación de Mercados, Gerencia y Comunicación Corporativa. Formó y dirigió durante 9 años el Departamento de Marketing y Publicidad de la Universidad Técnica Particular de Loja. Desde noviembre de 2007 es Director Nacional de Educación Continua de la UTPL. 
deseados soportes publicitarios que están siendo considerados cada vez más por los anunciantes en el momento de la planificación de las estrategias a fin de alcanzar sus objetivos. Estas transformaciones obligan a los responsables de comunicación de las marcas, a realizar un replanteamiento en el uso integral de los medios de los que disponen.

El presente análisis tiene como objeto conocer cómo las empresas ecuatorianas están manejando la marca a través de una integración de medios, con los canales offline y online como son las redes sociales.

Se aplica la técnica de estudio de casos a las campañas publicitarias de organizaciones representativas de tres diferentes sectores económicos. Para el levantamiento de información se realizaron entrevistas semiestructuradas a los responsables de esas campañas, tanto de las empresas, como de sus agencias de publicidad digital especializadas. Se aplicaron además entrevistas estructuradas a agencias de publicidad digital y tradicional en Ecuador y a expertos nacionales e internacionales con alta experiencia en la academia, en planificación, consultoría y capacitación en temas de marca, marketing digital, redes sociales y otros en desarrollo de campañas.

A la luz de los resultados, se observa, que es muy creciente la inclusión del canal online al mix de medios, por parte de los gerentes de mercadeo, por lo que ya no se considera una improvisación. La enorme penetración de Internet en Ecuador, que alcanza el 37,3\%, según datos del INEC, presenta grandes oportunidades a las marcas a través del uso de estrategias publicitarias atractivas para llegar a sus nichos de mercado precisos. Una clara muestra de ello es que el $64 \%$ de las agencias sondeadas, todas las digitales y una parte considerable de las tradicionales, incluyen Facebook en las campañas de sus clientes. Además un $59 \%$ de dichas agencias consideran que las acciones comerciales deben apostar por una campaña integral.

Palabras claves: Marca online, integración, redes sociales. 


\begin{abstract}
ON LINE BRANDING AS PART OF INTEGRAL COMMUNICATION. MANAGEMENT OF ECUADORIAN BRANDS IN SOCIAL NETWORKS
\end{abstract}

The use of Internet as publicity and advertising has a wide reach. A clear example is the advertisers demand on spaces as ranking of the words that are most looked for in Google. Social networks on line become wanted advertising supports that are every day more considered by announcers when planning strategies. These transformations force the communicators to rethink the integral use of media.

The present analysis has as a target to know how Ecuadorian business is managing brands through media integration in on line and off line channels.

The methodology employed is the study of cases of the advertising campaigns of representative organizations of three different economy sectors. Data was gathered through semi- structured interviews to the responsible people for those campaigns both in business and in agencies. Other polls were applied to digital and traditional agencies of Ecuador and national and international experts.

The results show that the use of the on line channel increase in the media mix, for it is no longer considered an improvisation. The wide penetration of Internet in Ecuador (a 37,3\%, according to the INEC) presents huge opportunities to brands. A sample is that the $64 \%$ of the analyzed agencies include Facebook in their clients campaign. Besides, a $59 \%$ of those agencies consider commercial actions must strive for an integral campaign.

Keywords: on line brands, integration, social networks

\title{
Resumo:
}


O uso da Internet na promoção e publicidade tem um escopo amplo. Como observado Araceli Castelló (2010, p. 14-15) um exemplo claro é a demanda de anunciantes, como espaços de palavras mais procurado ranking no Google. Além disso, as redes sociais online tornam-se meios de publicidade não desejados estão sendo cada vez mais considerado pelos anunciantes no planejamento de estratégias para atingir seus objetivos. Estas transformações exigem fabricantes de comunicação da marca, para realizar uma abrangente repensar o uso dos meios à sua disposição.

Esta análise tem como objetivo saber como as empresas estão a gerir a marca equatoriana através de integração de mídia com canais offline e online, como redes sociais.

Técnica é aplicada a um estudo de caso das campanhas publicitárias de três organizações que representam os diferentes sectores económicos. Para reunir informações sobre entrevistas semi-estruturadas foram responsáveis por estas campanhas, as duas empresas e suas agências de publicidade especializada digital. As entrevistas estruturadas foram aplicadas, além de agências de publicidade digital e tradicional no Equador e especialistas internacionais com vasta experiência em academia, consultoria, planejamento e treinamento em branding, marketing digital, redes sociais e outras campanhas em desenvolvimento.

Em função dos resultados, observa-se que a inclusão é crescente o canal online para misturar mídia, gerentes de marketing, de modo que não é mais considerado uma improvisação. A penetração da Internet enorme no Equador, que chegou a $37,3 \%$, de acordo com o INEC, apresenta grandes oportunidades para as marcas através do uso de estratégias de publicidade atraentes para alcançar seu nicho preciso. Um exemplo claro é que 64\% das agências pesquisadas, totalmente digital e uma parte considerável do tradicional, incluem Facebook em campanhas cliente. Além disso 59\% das agências considerar ações comerciais devem se concentrar em uma campanha integrada. 
Palavras-chave: Marca on-line, integração, redes sociais.

Año VI, Número 10, (2013), v1 pp. 95-116 http://www.redmarka.org/ 


\section{Introducción}

La Web 2.0 encierra aquellas plataformas que implican la interacción y la personalización, el intercambio y la colaboración, son espacios comunicativos relacionales, en los que se elabora y se coopera como redes sociales, blogs, wikis, etc. En este contexto la comunicación comercial cada vez gana espacio y busca la manera de introducirse de forma más sutil, al considerar la personalización de esos medios. Es así que, cuando se habla de la evolución en la manera de comunicar la marca, se hace referencia a la interactividad que, en la red digital, viene a ser la base de todo el proceso de su creación. En la implantación, desarrollo y asentamiento de este proceso, es importante conocer lo que el público piensa de la organización y cómo está ubicada la firma ante ellos. Por lo tanto, cuando se habla de una marca posicionada, se hace referencia a una gestión integral en su comunicación, que es la suma de todos sus procesos comunicativos.

\section{La marca online}

De cara a los vertiginosos cambios en el campo ocupacional, los gestores de marketing intentan sacar provecho a los beneficios que ofrecen las redes sociales. En efecto, el experto ecuatoriano en este campo, Christian Espinosa destaca que: al ser utilizadas en forma efectiva, éstas pueden convertirse en un instrumento fundamental para que las empresas se conecten con sus seguidores y clientes, y puedan conocer sus hábitos de compra, tendencias del mercado, etc.: "Si se las usa de forma efectiva, y la marca se convierte en una parte real con un grado de reputación en la red social, los beneficios corporativos son mayores que cualquier otra iniciativa, más tradicional de marketing que se pueda pensar" (Espinosa, 2010, p. 18). Por lo tanto, las nuevas herramientas Web 2.0 enriquecen la forma en que las marcas toman contacto con sus targets. Esto requiere de un mayor conocimiento tanto de los hábitos de consumo y de los soportes a través de los cuales se realiza dicho contacto. Pero los hábitos de consumo de productos y servicios de los 
consumidores son cambiantes, y ello depende de la presencia de nuevos productos, de los nuevos usos que se les da, de nuevas formas de comunicar, de la renovación constante de las tecnologías, etc. Todo ello exige que los anunciantes tengan una actualización constante sobre dichos hábitos.

Según Castelló, las nuevas tecnologías sociales dan paso a la interacción social que cada vez influye más en las decisiones de compra de los consumidores, así como en la imagen de las empresas: "Escuchar, conversar y relacionarse con los clientes a través de las nuevas tecnologías supone una transformación total de la cultura empresarial” (Castelló, 2010, p. 15), porque el consumidor, al convertirse en distribuidor de contenido, cumple un rol importante en las estrategias comerciales, ya que interviene en los mensajes publicitarios, ya sea en su contenido como en su recorrido, y esto se debe a la viralidad que le permite interactuar constantemente con las marcas (o, cit., 1516).

Las marcas cumplen con una misma función en el campo analógico y digital, que es la de diferenciar y transmitir valores, identidad e imagen. Sin embargo hay lineamientos específicos en su uso online. Los responsables de marketing cada vez más asignan un monto a campañas digitales, aunque se inicien probando y cometiendo errores. No es sorprendente ver que empiecen utilizando los mismos formatos diseñados para otros medios como por ejemplo folletos convertidos en pdf, pero esto resulta admisible en una primera intención. En un ambiente virtual el material debe estar en formato multimedia e interactivo, y si lo gestionan en forma adecuada, las marcas pueden tener mayor oportunidad por la relación directa y afectiva que se da a través de la interacción:

"Las marcas, ahora, no se crean simplemente manipulando enunciados icónicos o lingüísticos: también la experiencia interactiva se convierte en un elemento fundamental de su proceso de construcción" (Capriotti, 2007, p. 65). Éstas, tarde o temprano, terminan en Internet y teniendo su misma identidad y forma, involucran al usuario en un ambiente dinámico e interactivo. Quizás esta sea una marcada diferencia entre la marca analógica y la digital. 
La empresa, al gestionar todos sus intangibles a través de la comunicación de la marca, intenta crear un valor en su relación con el target. Ya no son suficientes los circuitos de atención al cliente como el call center, o el servicio al cliente en los bancos por ejemplo. El prospecto necesita de una atención personalizada y ésta es una gestión de la empresa:

"Llegar al usuario a través de nuevos canales que permiten una relación más cercana entre target y marca, como son los blogs y las redes sociales online, canales que además permiten una mayor afinidad y personalización del mensaje, gracias a las posibilidades de segmentación... Las marcas deben desarrollar la capacidad de innovar a través del establecimiento de relaciones y de un diálogo con él” (Castelló, 2010, p. 37).

El poder de segmentación o microsegmentación que tienen los medios sociales, son sin lugar a duda, una ventaja que marca una diferencia con el marketing tradicional. Para José Luis Gill, ahora los consumidores se han "tecnosofistificado" y se evidencia sobre todo en los jóvenes a los que la marca se aproxima pero formando parte de su estilo de vida y de sus preferencias y usando canales frescos que les aporten contenido, y especialmente contextualizados en su forma de vida (Gill, 2010).

Cada vez, los gestores de la comunicación comercial observan contenidos de otras marcas, lo que genera interés en los medios online. Si los consumidores se están convirtiendo en usuarios activos de estas herramientas, es ahí donde se debe hacer presencia de marca, pero con propuestas que generen valor. Una marca tiene la capacidad de singularizar a un producto, de iguales. Como afirma el Dr. Jesús Timoteo Álvarez profesor de la Universidad Complutense de Madrid: "Son las marcas quienes animan a la gente y les infunden esperanza, pasión por algo, recuerdo y memoria de conceptos morales, fe en la vida y en la acción" (Álvarez, 2009, p. 28). Las marcas "representan toda la percepción que el cliente tiene de una empresa; es la imagen, la emoción o el mensaje que la gente recibe cuando piensa en esa empresa o sus productos" (Iruzubieta, 2010). Es lo que la gente de marketing llama, "enamorarse de la marca" y los espacios sociales son los que permiten crear una conexión emocional con ellas 
pero cuando se establece una diferencia: Las marcas "construyen un significado al proponer una diferencia" (Capriotti, 2007, p. 61).

\subsection{Del branding al ebranding}

Ante lo expuesto, Castelló (2010, p. 36) considera que el branding se relaciona directamente con las actitudes de los individuos, dependiendo de la imagen que se genera en el receptor, por lo tanto los objetivos publicitarios deben responder en función del nivel actitudinal del destinatario en el que se desea influir. El branding (que permite la asociación de ideas y la predisposición favorable hacia la marca) hace posible una diferenciación que se logra por la gestión de todos los intangibles, y esto se da cuando se llega al usuario a través de nuevos canales que posibilitan una relación mucho más cercana, tales como los blogs y las redes sociales. A su vez estas herramientas hacen que haya una mayor afinidad y personalización del mensaje, sobre todo por las posibilidades de segmentación.

Algunos autores de teorías digitales como Castelló lo siguen llamando branding, pero en este capítulo se refiere como eBranding; este fenómeno ha existido desde siempre, Internet solo es uno de los medios más nuevos que permite llevar la identidad de una organización a sus públicos en cuestión de segundos. Por lo tanto, branding es igual que eBranding sea offline u online. La esencia por sí misma no cambia tanto, pero el enfoque sí, ya que Internet es un excelente vínculo entre marca y consumidor por su rapidez y eficacia:

"El eBranding es el proceso de transformar un sitio web en un experiencia única para el usuario. Única por la calidad de su diseño, por la utilidad y usabilidad de sus contenidos y por la simple y eficaz lógica funcional en la navegación e interacción con dicho sitio web" (Vallet, 2005, p. 33).

La web 2.0 ha permitido desarrollar aspectos fundamentales del marketing: "obtener una marca fuerte y una relación sólida con el cliente" (Ros, 2008, p. 103). Es un nuevo contexto en donde se potencia la interacción, se establecen contactos, es posible participar en las conversaciones, etc. Al mismo tiempo se habla de un nuevo reto: "la democratización del mundo de las marcas". 
En torno a este concepto se resume algunas ventajas; entre las principales, Araceli Castelló expone:

"Capacidad de reacción inmediata en la gestión de la imagen y la comunicación corporativa, capacidad de convocatoria/creación de comunidades, cobertura, integración de la marca, mejora de la notoriedad e imagen de marca y cercanía de marca/marca amigable. Las opiniones de los usuarios de las comunidades online facilitan la actualización y mejora de la imagen corporativa y las marcas deben generar una reputación 2.0 en un entorno de colaboración y cooperación con el consumidor" (Castelló, 2010, p. 107).

Claro que también se puede correr el riesgo de que no todos los comentarios sean favorables hacia la marca, sin embargo esto dependerá de su adecuada gestión integral que incluye a los intangibles. Las marcas buscan ubicarse en la mente como únicas a través de una propuesta de valor para los usuarios, y tienen que estar conectadas a las tecnologías, pero sobre todo que los consumidores lo noten. Las firmas deben estar dentro de estas nuevas formas de comunicar, deben ir a la par con las innovaciones y no sólo limitarse a la publicidad masiva. El eBranding, debe generar en los usuarios vínculos emocionales hacia la marca. A medida que se va avanzando a esta consolidación de una nueva forma de vida, no está demás afirmar que el eBranding no es únicamente la presencia de la marca en las redes sociales a través del logo y contenidos. La construcción de una marca 2.0 tiene en cuenta aspectos que tienen que ver con la totalidad de la relación que necesariamente se debe construir con los clientes.

\subsection{Posicionamiento de la marca en redes sociales}

El posicionamiento es una de las tareas más complicadas para las organizaciones. Pero este no es un concepto de Internet, sino que se encuentra en el ámbito del Marketing y la Publicidad. Aunque la Publicidad, hasta cierto punto, tiene una resistencia debido a su naturaleza misma de propiciar la compra/venta o el intercambio de servicios, se constituye en una 
necesidad ineludible para cualquier organización, sea pública o privada, para poder obtener beneficios (Dans, 2010, pp. 273-274). En este sentido, la empresa puede contar con el mejor producto y/o servicio, pero si no lo publicita de cualquier forma, no llegará muy lejos. La meta de las organizaciones, a través de la promoción de sus productos, es alcanzar una posición privilegiada en el mercado. Existen grandes marcas que ya han conseguido un alto posicionamiento, y se podría pensar que no necesitan más publicidad, sin embargo siguen asignando grandes sumas de dinero a esta nueva herramienta como es Internet.

Un producto debe tener un posicionamiento efectivo para que lo puedan referir, entonces resulta clave trabajar primeramente en el posicionamiento de marca, para que luego pueda ser recomendado. Resultaría interesante revisar los resultados que se pueden obtener a través de mediciones en investigaciones de mercado. Las herramientas digitales constituyen un excelente recurso para ubicar a un producto y/o servicio en la mente del consumidor o posible usuario. En Internet, si se logra que la marca aparezca en los primeros lugares de los buscadores más importantes, se tendrá asegurado un alto número de visitas diarias. A esto también se suma un efecto multiplicador alto: "En la época del Viral Marketing un usuario satisfecho que le "pase el dato" a un amigo o colega vale más que un spot. El boca a boca hará el resto" (Capriotti, 2007, p. 71).

En este mismo contexto Boronat \& Pallares (2009, p. 22) afirman que el problema para las organizaciones no está en el desconocimiento del manejo de su propuesta de valor o posicionamiento en la Red, sino en definir comunicacionalmente cuáles son sus ejes diferenciadores 0 su posicionamiento deseado. El problema es comunicativo: "Sin un importante trabajo comunicacional previo a Internet, difícilmente sabremos trasladar a la Red un posicionamiento claro y contundente" (o, cit., 22). Desde esta perspectiva, es recomendable que una marca tenga un camino recorrido y un trabajo comunicacional estructurado bajo aspectos que lo distingan de sus competidores; y claro, soportado en medios tradicionales, con una credibilidad y reputación ganada, para luego complementar su estrategia de comunicación con medios digitales. 


\section{Comunicación integral en digital}

Solo se debe revisar los datos del crecimiento progresivo de las audiencias de lectores de medios digitales para ratificar que los cambios ya se están dando y de forma acelerada. Este cambio de mentalidad en la forma de comunicarse con los medios, también se ha visto reflejado en la comunicación interna que los directivos mantienen con los empleados de sus empresas: "La comunicación ya no es lineal, de arriba abajo, sino que se fomenta una conversación entre todos los empleados, un modelo más participativo y que permite tener flujos comunicativos descendentes: de arriba a abajo, y ascendentes: de abajo a arriba" (Celaya \& Herrera, 2007, p. 39). Por lo tanto, en este modelo de comunicación participativa no hay una sola dirección, sino que todos forman parte del mensaje; lo reciben en forma conjunta y lo remiten, creando conversaciones participativas. La comunicación integrada involucra a todos los empleados que son parte de la organización, pero, para el efecto, todos deben estar alineados a una identidad corporativa (misión, valor) que es transmitida a los diferentes públicos. Además todos deben estar actualizados a fin de que puedan propiciar información en sus ámbitos diversos: comercial e institucional. Quién mejor que los propios empleados para trasladar la esencia de la organización.

Por otro lado, si bien Internet presenta ventajas propias que lo diferencian de otras formas de comunicar; no se puede perder de vista la total conveniencia de apoyar la comunicación sobre un pilar de integridad, que sume los esfuerzos de los distintos medios y que pueda alinear sus resultados. Es así que cada vez las campañas combinan más estrategias offline y online, por lo tanto se hablaría de una comunicación integrada. Resulta indispensable gestionar campañas de marketing que incluyan componentes digitales y tradicionales. Para Félix Cuesta y Manuel Coto $(2010$, p. 135) las mal llamadas agencias de $360^{\circ}$ distan mucho de estar regidas por una estrategia verdaderamente global. Las campañas realmente integradas son un nuevo icono en este nuevo marketing, y se las conoce como blended marketing, en donde ya no sólo se trata de mejorar a las campañas tradicionales con refuerzos digitales haciéndolas "blended", sino que busca hacer un marketing 
más directo y más pull gracias a los canales online. Dicho en palabras de Manuel Coto:

"Es clave integrar estas nuevas posibilidades de marketing digital con los formatos y medios que llevan años demostrando su eficacia. De hecho, el uso combinado de herramientas online y offline en una misma campaña de marketing, potencia el recuerdo de los valores de marca en hasta un $18 \%$, lo que refrenda a Internet como un medio muy rentable e inexcusable pero en ningún caso exclusivo para toda campaña de marketing, comunicación y publicidad" (Coto, 2008, p. 5).

En un artículo, Araceli Castelló (2012) indica que en el 2007 la publicación Advertising Age avecinaba que las fronteras del marketing entre los formatos digitales y los tradicionales se difuminarán irremediablemente a lo largo de los siguientes cinco años, por que el plan de marketing actual es una combinación que refuerza los valores de marca y debe ser digital porque integra a las herramientas online con formatos y medios tradicionales.

Hay acciones online, que terminan incidiendo en el offline. Trasladando esta idea, de Gabriel Eroles (2010, p. 34), existen campañas en donde la gestión online permite potenciar a las actividades tradicionales, por ejemplo: el tráfico que se puede provocar para citar algún evento o las redes sociales como canal de información. La micro segmentación de las redes puede permitir la filtración de bases de datos para el envío de una carta de presentación o un catálogo. El online puede ser el vehículo para materializar acciones de participación o concursos que se llevan a cabo en el offline. Al tomar a los medios como un todo, es importante romper cualquier barrera que separe las estrategias en línea y fuera de línea: "Si se logra de forma óptima que el impacto de las acciones presenciales se exporte automáticamente al online -estimulando a los usuarios para registrarse en la web, por ejemplo- se consiguen resultados imbatibles" (o. cit., 37). También hay acciones online que requieren del soporte tradicional. Por ejemplo, juegos virales online que necesitan de folletería, anuncios en prensa, publicidad exterior, para convocar a una mayor cantidad de público. 
Al hablar de un marketing integrado, Burgos \& Cortés (2009, pp. 32-33) hacen alusión a un marketing multicanal, en función de las nuevas tipologías e innovaciones para la elaboración de campañas. Las organizaciones combinan acciones de mercadeo directo online; de igual manera el Mobile marketing y los social media: las empresas pequeñas disponen de blogs, RSS y redes sociales. De todos los estudios y cifras se puede asegurar que la integración de campañas es una realidad; es más, la tendencia va hacia una mayor focalización en acciones on-line versus off-line en el mix de marketing. Sin embargo, quien define en dónde se debe centrar una organización no es el mercado ni las tendencias, sino los clientes y prospectos. Tocaría pues escuchar en qué, cómo, y a través de qué medios quieren ser contactados, así como las propuestas de valor que ellos consideran importantes. Sólo así se podrá elaborar un oportuno mix de acciones para captación y fidelización.

El responsable de la gestión comunicacional debe procurar en lo posible una comunicación integral, dentro del planteamiento estratégico o la estrategia global de comunicación; una función coordinada de medios online y offline como: La técnica publicitaria «Below The Line» (BTL), relaciones públicas, mercadeo directo, merchandising y otros. Es decir que al online le corresponde formar parte del mix de medios como cualquier otro medio, y en cada uno de ellos seleccionar los soportes más apropiados que permitan el cumplimiento de objetivos comunicacionales. Pero todo dependerá del tipo de producto, de los objetivos de la organización y del grupo objetivo al que se quiere dirigir el mensaje, porque pueden existir campañas que únicamente requieran de soporte digital o solo acciones tradicionales.

\section{Resultados}

Del análisis cualitativo aplicado a empresas de tres diferentes sectores económicos: educativo (Universidad Técnica Particular de Loja), de servicios (Cadena de supermercados Supermaxi) y comercial (Automotores Chevrolet), se conoce los siguientes resultados: 
visitas, interacción, viralización y exposición de la marca. Pero, dentro de su categoría, la inversión en digital es alta.

Dentro de su planificación estratégica de medios, las tres empresas integran medios masivos como principales, pero también los utilizan para soportar a las campañas digitales. Pese a que las tres organizaciones tienen objetivos diferentes, hacen uso de las redes sociales porque les permite una alta exposición de la marca, y esto se refleja en la cantidad de visitas y registros. No todas las organizaciones implementan su fan page con fines comerciales, incluso los propios seguidores sólo pueden buscar información en general.

La globalización conlleva un proceso de integraciones empresariales en todos los niveles, incluso con extensión internacional. A medida que un sector económico se va consolidando más, se ve influenciado por las tendencias económicas y de empresa a nivel mundial. Tal es el caso de Ecuador que ha ido madurando gradualmente en cuanto al desarrollo del sector publicitario. Esto se puede observar en el incremento de numerosos oferentes con estándares diversos de servicios, así como la acrecentada evolución de la demanda, por lo que agencias extranjeras han implementado sucursales en este país y, a su vez, las agencias ecuatorianas han buscado establecer sociedades con empresas multinacionales de publicidad. Esto les ha permitido crecer en el mercado, y ampliar su portafolio de clientes. Y en esta misma línea se suman la aparición en el país de otras agencias que dan soporte a la actividad comercial, tal como las de relaciones públicas, de "Customer Relationship Management» (CRM), BTL, etc.

Con este preámbulo, se evidencia que las grandes organizaciones, sobre todo las que tienen fines netamente comerciales se sirven de los servicios no sólo de agencias publicitarias tradicionales y digitales, sino de los diferentes tipos de agencias, pese a que cuentan con su propio departamento de mercadeo, tal es el caso de las empresas en estudio. Su liderazgo en el mercado, ratifica su posicionamiento, sobre todo por ser organizaciones que desarrollan actividades de responsabilidad social. 


\begin{tabular}{|l|}
\hline La marca desde el punto de vista de gestores de agencias tradicionales, \\
versus digitales \\
\hline $\begin{array}{l}\text { Las agencias digitales tienen mayor cantidad de indicadores que las } \\
\text { tradicionales, pero los resultados provocan inquietud. }\end{array}$ \\
$\begin{array}{l}\text { El } 64 \% \text { de las agencias, entre ellas todas las digitales incluyen redes sociales } \\
\text { y en las tradicionales sigue siendo bajo }\end{array}$ \\
\hline
\end{tabular}

Es muy importante medir la ejecución del plan de medios y cuál es el ciclo de conversiones de la página. Todos esos datos permiten el cambio o implementación de nuevas estrategias. Si bien existen algunos instrumentos de medición de la efectividad de las campañas digitales, causan de alguna manera inquietud en los gerentes de agencias tradicionales ya que, hasta cierto punto, existe desconfianza en los resultados presentados.

La inclusión del canal online al mix de medios está creciendo rápidamente, por lo que ya no se considera una improvisación. La enorme penetración de Internet en Ecuador, que alcanza en las zonas urbanas el 37,3\%, según datos del Instituto Nacional de Estadística y Censos de Ecuador (INEC) de 2010, presenta grandes oportunidades a las marcas a través del uso de estrategias publicitarias atractivas para llegar a sus nichos de mercado precisos. Una clara muestra de ello es que el $64 \%$ las agencias sondeadas, todas las digitales y una parte considerable de las tradicionales, incluyen Facebook en las campañas de sus clientes, entre el $50 \%$ y el $100 \%$. Sin embargo no en todas las agencias tradicionales se realizan actividades relacionadas con esta red social; de las 12 agencias (de 14 que existen en total) que dieron respuesta, en 7, la mayoría de sus clientes integran estas acciones y en 5 la inclusión aún es baja. Se debe considerar que cada agencia lleva una gran cantidad de cuentas, por lo tanto en este proceso, que avanza a pasos agigantados, les corresponde a éstas, evangelizar a las organizaciones sobre el uso y optimización de las tecnologías digitales.

\section{La marca desde el punto de vista de expertos-agencias nacionales, expertos internacionales y directores de cuentas $82 \%$ de expertos, $50 \%$ gerentes de agencias y directores de cuenta de las}


organizaciones consideran que una marca no requiere de un posicionamiento previo para incursionar en las redes sociales.

No todas las agencias apuestan por una campaña integral (59\%).

Sólo el $29 \%$ de las agencias cuentan con su propio departamento digital.

El $43 \%$ de las marcas consideran que los resultados son excelentes.

El $82 \%$ de los expertos, el $50 \%$ de los gerentes de las agencias, y los directores de cuenta de las tres organizaciones en estudio consideran que una marca (tradicional no digital), no necesariamente debe tener un posicionamiento previo en medios tradicionales para que pueda ser introducida en las redes sociales. Existen ejemplos de marcas como Amazon o Google, que han cambiado los procesos tradicionales de construcción de marcas. Incluso hay firmas como Yahoo que han logrado un alto posicionamiento sin tener que reforzarse en piezas de medios tradicionales. Los profesionales que ejecutan acciones tradicionales y digitales consideran en un 59\% que las campañas publicitarias deben apostar por una atención integral, cosa que llama la atención.

El canal digital ha cambiado el concepto de ofrecer servicios de comunicación integral por parte de las agencias tradicionales, que a medida de las exigencias de las marcas, se ven en la obligación o de integrar un área digital a su agencia o de enlazar a los clientes con agencias digitales especializadas. De las 14 agencias tradicionales entrevistadas, solo cuatro tienen su propio departamento digital. Esto responde a la realidad en el ámbito publicitario: la baja inversión publicitaria online por parte de las empresas, el desconocimiento de las ventajas de medios online, el cambio que implicaría en la estructura organizacional de la agencia, la especialización, el incremento de los presupuestos para usar una comunicación de $360^{\circ}$, etc., han sido un tope para que las agencias tradicionales incursionen en el ámbito digital.

Como en toda gestión de publicidad, los resultados pueden ser excelentes o regulares dependiendo de la estrategia, la ejecución y el seguimiento que se haga. De pronto, para los responsables de la marca de UTPL y Supermaxi los resultados son calificados como buenos por las cifras en registros y por inconvenientes en el funcionamiento de la parte técnica del concurso o en la 
adquisición de las tarjetas. Y en sí, en digital se pueden presentar algunos factores que causen inconformidad. De las 21 agencias entrevistadas sobre los resultados de uso del Facebook en las campañas ejecutadas, el $43 \%$ las consideran excelentes (clientes satisfechos en resultados y operatividad), el $47 \%$ buenos (clientes satisfechos pero con alguna inconformidad) y el $10 \%$ no responden.

\section{Conclusiones}

Según el criterio de un número significativo de expertos y operativos de campañas se espera un futuro bastante alentador para las empresas en el uso de esta red social que ya que se ha convertido en el principal espacio para la construcción de la marca, fidelización y, en muchos casos, venta y atención al cliente. Sin embargo, dado el crecimiento de inversión por año en Internet, al momento en Ecuador, las organizaciones pese a que ya se encuentran haciendo alguna actividad online, aún no se arriesgan a tener una mayor inversión en campañas digitales en Facebook, porque la actividad digital está en crecimiento así como el nivel de conocimiento de las acciones aplicables.

Como ya se señaló en Ecuador, cada vez se incrementa el porcentaje de marcas que incluyen en su plan estratégico campañas en medios digitales. Como un indicador, en el país se realizan de forma constante evaluaciones de marca. En el 2011, la Corporación Ekos, cuya revista se ha posicionado como la mejor en negocios de Ecuador, premió a las empresas con mayor capital de marca del Ecuador, tomando como modelo la metodología Brand Equity Index (BEI), desarrollada por la consultora chilena de Praxis y auditado por Deloitte Touche. Este modelo se basa en cuatro pilares que se constituyen en el fundamento de la conexión entre la marca y el comprador: Energía, Visibilidad, Bonding (compromiso del cliente con la marca) y Valores o Atributos de Marca. En total, 26 marcas en 21 sectores con mayor predominancia en el país, de las cuales ocupan el primer lugar, cada una en su sector y categoría, dos de las empresas en estudio: Supermaxi y Chevrolet. Como dato adicional en la investigación se consultó a través de las agencias de publicidad tradicionales y digitales y, bajo una verificación en Facebook que si estas marcas poseían fan 
page. Se encontró que de este total, el $88 \%$ incursionan en estrategias digitales, por lo tanto las marcas líderes en el mercado establecen la diferencia a través de estrategias on y offline.

Son muy pocas las agencias que poseen un departamento de comunicación digital, pues éstas deben seguir y ser fuertes en estrategias creativas, diseño, planeación de medios tradicionales y otras formas de comunicar, y dar paso a las agencias digitales especializadas, que cada vez más seguirán surgiendo, a medida que vaya incrementándose la penetración de Internet y la demanda de las organizaciones.

Ante la satisfacción por los resultados de las campañas digitales, un compromiso para las organizaciones sería evitar compararse con otras marcas y querer obtener iguales resultados puesto que cada organización tiene su propia identidad corporativa. Además que se requiere que se comprometan con el canal ya que el desarrollo de una comunidad es muy rentable pero requiere de tiempo y recursos. Y para las agencias, pese a que su intención es siempre ofrecer campañas altamente creativas, pero también deben tomar en cuenta las implicaciones técnicas, de tiempo y de complejidad en el uso de la aplicación y con los correctivos necesarios, perfeccionar la estrategia.

Esta investigación logra disuadir a ciertos discursos "extincionistas" que están de moda. La aparición de un nuevo medio, no debe significar que otro deba desaparecer. Internet no va a reemplazar a los medios tradicionales, sino que formará parte integral de cualquier campaña.

Pese a las debilidades que pueda presentar esta red social, es importante considerar el abanico de posibilidades que ofrece. Lo más probable es que de aquí a otros años vendrán nuevas redes, pero hoy en día y de cara al futuro es una apuesta sólida y no sólo tiene que ver con el crecimiento o el número de usuarios, sino en el crecimiento que tiene como plataforma, en las opciones que da para comunicar, en estrategias, en desarrollos, en como aporta esta red al CRM de una marca.

\section{Bibliografía}


Álvarez, J. (2009). Senderos en el bosque: Condicionantes y perspectivas de las estrategias de comunicación en la sociedad red, en Publicidad y comunicación corporativa en la era digital. Ramos, Marina; Garrido, Manuel; Rodríguez, Juan Carlos (Coords.). Madrid: Pirámide (Grupo Anaya, S.A.).

Boronat, D., \& Pallares, E. (2009). Vender más en Internet. Barcelona: Gestión 2000.

Burgos, E., \& Cortés, M. (2009). Iníciate en el marketing 2.0. Los social media como herramientas de fidelización de clientes. España: Gesbiblo, S. L.

Capriotti, P. (2007). Gestión de la marca corporativa. Buenos Aires: La Crujía.

Castelló, A. (2010). Estrategias empresariales en la Web 2.0. Alicante: Editorial Club Universitario.

Castelló, A. (2012). LOS PORTALES VERTICALES DE MARKETING Y PUBLICIDAD EN LOS MEDIOS SOCIALES, [recuperado 19-112012] de Vivat Academia, № 117E: http://www.ucm.es/ info/vivataca/numeros/n117E/DATOSS.htm.

Celaya, J., \& Herrera, P. (2007). Comunicación empresarial 2.0. La función de las nuevas tecnologías sociales en la estrategia de comunicación empresarial. Barcelona: Grupo BPMO.

Coto, M. (2008). El plan de marketing digital. Blended Marketing como integración de acciones on y offline. Madrid: Pearson Educación S.A.

Cuesta, F., \& Coto, M. (2010). Marketing directo 2.0: Cómo vender más en un entorno digital. Barcelona: Gestión 2000, Grupo Planeta.

Dans, P. (2010). Internet. Edición 2010. Madrid: Anaya Multimedia.

De Gabriel Eroles, J. (2010). Internet Marketing 2.0. Captar y retener clientes en la Red. Barcelona: Reverté, S.A.

Espinosa, C. (2010). Las redes sociales y su influencia en la marca. Ekos, № $194,18$.

Gill, J. L. (2-03-2010). VENTAJAS DE LAS NUEVAS TECNOLOGÍAS APLICADAS A LAS ESTRATEGIAS DE COMUNICACIÓN DE LAS MARCAS, [recuperado 07-12-2012], de elpublicista.com: http://www. 
elpublicista.es/frontend/elpublicista/noticia.php?id_noticia=6540\&id_s eccion $=33$.

INEC (2011). ESTADÍSTICAS TIC'S 2010, [recuperado 22-01-2013] http://www.inec.gob.ec/estadisticas/index.php?option=com_remositor $y \&$ ltemid $=\&$ func $=$ select\&id $=93$ \&lang $=e s$

Iruzubieta, G. (2010). CUADERNOS DE COMUNICACIÓN INTERACTIVA. EL LIBRO BLANCO DE IAB. EL PANORAMA INTERACTIVO DE LOS GRUPOS DE PRENSA Y REVISTAS EN ESPAÑA (IAB Spain), Vol. $10 . \quad$ Obtenido de iabspain.net: http://www.iabspain.net/wpcontent/uploads/downloads/2012/02/10_L B_Medios_Comunicacion_Online.pdf.

Ros, V. (2008). Posiciona tu marca en la Red: e-Branding. La Coruña: Netbiblo, S.L.

Vallet, G. (21 de Junio de 2005). Tesis. eBranding. La creación de marca digital en la era de la conectividad. Barcelona: Universidad Autónoma de Barcelona, U.A.B. 